\title{
Immunohistochemical measurements of nerves and neuropeptides in diabetic skin: relationship to tests of neurological function
}

\author{
D.M. Levy ${ }^{1}$, G. Terenghi ${ }^{2}$, X.-H. Gu ${ }^{2}$, R.R. Abraham ${ }^{1}$, D. R. Springall ${ }^{2}$ and J.M.Polak ${ }^{2}$ \\ ${ }^{\iota}$ Department of Diabetes and Endocrinology, Central Middlesex Hospital \\ ${ }^{2}$ Department of Histochemistry, Royal Postgraduate Medical School, London, UK
}

\begin{abstract}
Summary. Image-analysis was used to measure nerves immunoreactive to the general neuronal marker protein gene product 9.5 (PGP 9.5-IR) and the neuropeptides calcitonin generelated peptide and vasoactive intestinal polypeptide in standardised leg skin biopsies of three age-matched groups of young subjects: non-diabetic $(n=14)$, diabetic patients with normal small fibre function ("non-neuropathic", $(n=11)$ and diabetic patients with abnormal small fibre function ("neuropathic", $n=11$ ). Depletion of nerves and neuropeptides was most marked in the epidermis, where calcitonin gene-related peptide-immunoreactivity was more frequently absent than PGP 9.5-IR in diabetic patients. Epidermal PGP 9.5-IR nerve area and counts were reduced in neuropathic compared with normal subjects $(p<0.001)$, as were epidermal calcitonin gene-related peptide nerve counts $(p=0.003)$. Sweat gland PGP 9.5 and vasoactive intestinal polypeptide, which may be involved in sweat production, showed no diminution in diabetic patients (area: $p=0.160, p=0.372$ by ANOVA). Two diabetic patients showed elevated sweat gland PGP 9.5-IR and three had increased sweat gland va-
\end{abstract}

soactive intestinal polypeptide; this may represent nerve proliferation. In local sweat tests, acetylcholine-stimulated sweat output was associated with increased immunoreactivity, while the sympathetic skin response showed inverse correlations with immunoreactivity. There were no consistent changes with other commonly-used neurophysiological tests. $\mathrm{HbA}_{1}$ correlated negatively with immunohistochemical measurements. Neuropeptide changes were seen in the absence of macro- and microvascular disease, and epidermal nerve depletion occurred in patients with normal thermal thresholds and cardiac autonomic function. Immunohistochemical measurement of cutaneous nerves in skin biopsies is a practical method for assessing peripheral small fibres in diabetes, and one which could be repeated in longitudinal studies.

Key words: Diabetic neuropathy, small-fibre studies, neuropeptides, immunohistochemistry, neurophysiology, sudomotor function.
Abnormalities of small, unmyelinated cutaneous nerve fibres are thought to be responsible for the frequent occurrence of painful symptoms in diabetes mellitus [1], and to contribute to the development of neuropathic foot ulceration $[2,3]$. Some authors have considered these fibres to be the first affected in diabetic neuropathy [4]. They immunostain with a variety of antibodies to neuropeptides; for example, substance $P$ and calcitonin gene-related peptide (CGRP) localise to "sensory" C fibres subserving skin axon reflexes [5], while others, such as neuropeptide $Y$ and vasoactive intestinal polypeptide (VIP) are found in peripheral "autonomic" nerves supplying blood vessels and sweat glands respectively [6].

All mammalian nerves immunostain for general neuronal markers, including protein gene product 9.5 (PGP 9.5) $[7,8]$, and the cutaneous innervation can be demonstrated in its entirety by the use of antibodies to these mar- kers, in contrast to the sub-populations of neurons immunoreactive for specific neuropeptides.

In a previous study we reported that observer-graded assessments of PGP 9.5-immunoreactivity (IR) and IR to specific neuropeptides were reduced in diabetic skin, with more marked reduction in patients with overt neuropathy; however, in the population we studied, macrovascular disease may also have contributed to this reduction [9]. Lindberger et al. [10], using a semi-quantitative method, also found that substance P- and CGRP-IR were reduced in skin biopsies, even in diabetic patients with no clinical or neurophysiological evidence of neuropathy.

In this study we have taken elective skin biopsies from a standardised site and measured cutaneous nerves immunoreactive to PGP 9.5, CGRP and VIP by using a computerised image-analysis system. Three groups of agematched subjects, well characterised by quantitative tests 
Table 1. Clinical characteristics of the three groups of subjects. Results are given as mean (range). $p$ values are for Student's t-tests, and relate to comparisons between the non-neuropathic and neuropathic patients

\begin{tabular}{|c|c|c|c|c|}
\hline & Normal subjects & Non-neuropathic subjects & Neuropathic subjects & $p$ \\
\hline Age (years) & $28(19-58)$ & $33(20-51)$ & $44(21-53)$ & 0.2 \\
\hline Duration of diabetes (years) & & $14(8-30)$ & $19(5-34)$ & 0.3 \\
\hline Type 1:Type 2 & & $10: 1$ & $11: 0$ & \\
\hline $\mathrm{HbA}_{1}{ }^{\mathrm{a}}$ & & $10.9(8-18.6)$ & $12.9(7.9-17.8)$ & 0.2 \\
\hline
\end{tabular}

${ }^{a}$ Reference range $<8.0 \%$

${ }^{\mathrm{b}}$ Assessed by direct ophthalmoscopy

Table 2. Neurophysiological characteristics of the diabetic subjects studied. Data are presented as mean (range) [number of subjects studied]. $p$ values refer to Student's t-tests comparing the non-neuropathic and neuropathic diabetic patients

\begin{tabular}{|c|c|c|c|c|}
\hline & Reference range & Non-neuropathic subjects & Neuropathic subjects & $p$ \\
\hline Marstock warm threshold $\left({ }^{\circ} \mathrm{C}\right)$ & $<8.6$ & $4.7(3.4-7.4)$ & $12(5.5-15.8)$ & $<0.001$ \\
\hline Marstock cool threshold $\left({ }^{\circ} \mathrm{C}\right)$ & $<4.7$ & $3.0(1.8-4.6)$ & $9.1(4.2-15.3)$ & 0.001 \\
\hline Respiratory $R R$ variation (bpm) & (age related) & $22(10-27)$ & $7(4-30)$ & 0.004 \\
\hline Valsalva ratio & $>1.13$ & $1.73(1.09-2.13)$ & $1.27(1.19-1.58)$ & 0.03 \\
\hline $\operatorname{SSAP}(\mu V)$ & $>4.9$ & $12(7-20)[9]$ & $5(2-8)[6]$ & 0.002 \\
\hline
\end{tabular}

CPMCV, Common peroncal motor conduction velocity; SSAP, sural sensory action potential amplitude

for the presence or absence of small-fibre neuropathy, were studied. Immunohistochemical measurements were compared with a variety of neurophysiological tests, reflecting function in both large- and small fibres.

\section{Subjects, materials and methods}

Three groups of subjects were studied: Group 1 consisted of normal subjects $(n=14)$. None had current or previous neurological disease, and all had an alcohol intake of less than 15 units per week. Subjects taking medication or with a history of trauma to the legs were excluded. Group 2 consisted of diabetic subjects ("non-neuropathic", $n=11$ ) with normal small-fibre tests i. e. normal thermal sensation (Somedic Marstock warm threshold $\leq 8.6^{\circ} \mathrm{C}$ and cool threshold $\leq 4.7^{\circ} \mathrm{C}[11]$ ) and three normal age-related cardiovascular autonomic function tests (respiratory RR interval variation, Valsalva ratio and 30:15 ratio [12]). Group 3 consisted of diabetic subjects ("neuropathic", $n=11$ ), with evidence of small-fibre neuropathy i.e. all had a warm thermal threshold greater than $8.6^{\circ} \mathrm{C}$ or cool threshold greater than $4.7^{\circ} \mathrm{C}$, or both, and at least one abnormal age-related cardiovascular autonomic test. Clinical and neurophysiological characteristics of the subjects are given in Tables 1 and 2. Subjects hat o clinical evidence of peripheral vascular disease, and only one (a "neuropathic" patient) was a cigarette smoker.

The skin biopsy procedure was approved by the Ethics Committee of Parkside Health Authority and all subjects gave their informed consent.

\section{Other neurophysiological and sweat tests}

Diabetic subjects had other neurophysiological tests in addition to thermal threshold and autonomic function tests: biothesiometry, to measure vibration perception thresholds at the medial malleolus [13], and electrophysiology (common peroneal nerve conduction velocity, sural sensory action potential anplitude), using standard surface recording techniques. In addition, three tests of local sudomotor function were performed: (1) Activated sweatspot density was measured over a $4 \mathrm{~cm}^{2}$ area of the dorsolateral aspect of the foot [14]. Pilocarpine (1\%) was iontophoresed at $1.5 \mathrm{~mA}$ for $5 \mathrm{~min}$, and sweatspot prints made at $4 \mathrm{~min}$ intervals to $20 \mathrm{~min}$ with bromophenol blue indicator papers. Average sweatspot density (number per $\mathrm{cm}^{2}$ ) was calculated from photographs of the prints $(12-20 \mathrm{~min})$ which were subjected to computerised image-analysis (Quantimet 970; Leica, Cambridge, UK). (2) Dynamic acetylcholine-induced sweat output [15] was measured over a $1.7 \mathrm{~cm}^{2}$ area of the dorsum of the foot at the same site as the pilocarpine test for a period of $12 \mathrm{~min}$ after $1 \%$ acetylcholine iontophoresis, using a sensitive direct-reading sudorometer. (3) The amplitude and latency of the sympathetic skin response was measured at the dorsum of the foot using a modified method of Shahani et al. [16].

\section{Skin biopsy}

Full-thickness skin biopsies were taken with aseptic technique from the lateral aspect of the middle one-third of the right lower leg. A standard elliptical template $1.7 \mathrm{~cm}$ long, $0.6 \mathrm{~cm}$ wide was used to mark and orientate the skin, and plain $1 \%$ lignocaine infiltrated widely around the biopsy site. Specimens were fixed immediately after removal. Two or three sutures were used to close the skin, and were removed 14 days later.

One diabetic and one normal subject developed a wound infection and dehiscence; in both instances written instructions relating to wound care had not been followed. Antibiotics were given and uneventful healing occurred. In two other normal subjects, minor degrees of wound dehiscence occurred after removal of the sutures, without further complications. 
Table 3. Quantitative immunohistochemistry. Results are given as mean (range) and immunoreactive nerve areas as $\mathrm{mm}^{2} \times 10^{-6}$

\begin{tabular}{|c|c|c|c|c|}
\hline & & Normal subjects & Non-neuropathic subjects & Neuropathic subjects \\
\hline \multicolumn{5}{|c|}{ Epidermis } \\
\hline PGP & $\begin{array}{l}\text { area } \\
\text { count }\end{array}$ & $\begin{array}{c}1020(522-2192) \\
15(8-24)\end{array}$ & $\begin{array}{l}843(196-1665) \\
11(5-17)\end{array}$ & $\begin{array}{c}391(0-992) \\
5(0-12)\end{array}$ \\
\hline CGRP & $\begin{array}{l}\text { area } \\
\text { count }\end{array}$ & $\begin{array}{c}282(0-957) \\
6(0-21)\end{array}$ & $\begin{array}{c}235(0-784) \\
4(0-12)\end{array}$ & $\begin{array}{c}57(0-268) \\
1(0-6)\end{array}$ \\
\hline \multicolumn{5}{|l|}{ Dermis } \\
\hline CGRP & $\begin{array}{l}\text { area } \\
\text { count }\end{array}$ & $\begin{array}{c}185(0-710) \\
3(0-8)\end{array}$ & $\begin{array}{c}270(0-931) \\
5(0-18)\end{array}$ & $\begin{array}{c}171(0-590) \\
2(0-8)\end{array}$ \\
\hline \multicolumn{5}{|c|}{ Sweat glands } \\
\hline PGP & $\begin{array}{l}\text { area } \\
\text { count }\end{array}$ & $\begin{array}{c}2403(941-4337) \\
41(23-83)\end{array}$ & $\begin{array}{c}1857(200-6320) \\
26(0-62)\end{array}$ & $\begin{array}{c}1554(0-5318) \\
23(2-53)\end{array}$ \\
\hline
\end{tabular}

PGP, Protein gene product 9.5; CGRP, calcitonin gene-related peptide; VIP, vasoactive intestinal polypeptide

Table 4. Statistical analysis of quantitative immunocytochemistry. Kruskal-Wallis analysis of variance was performed on the logarithmicallytransformed data; if significant $(p<0.05)$, unpaired $t$-tests were performed across the three pairs of groups

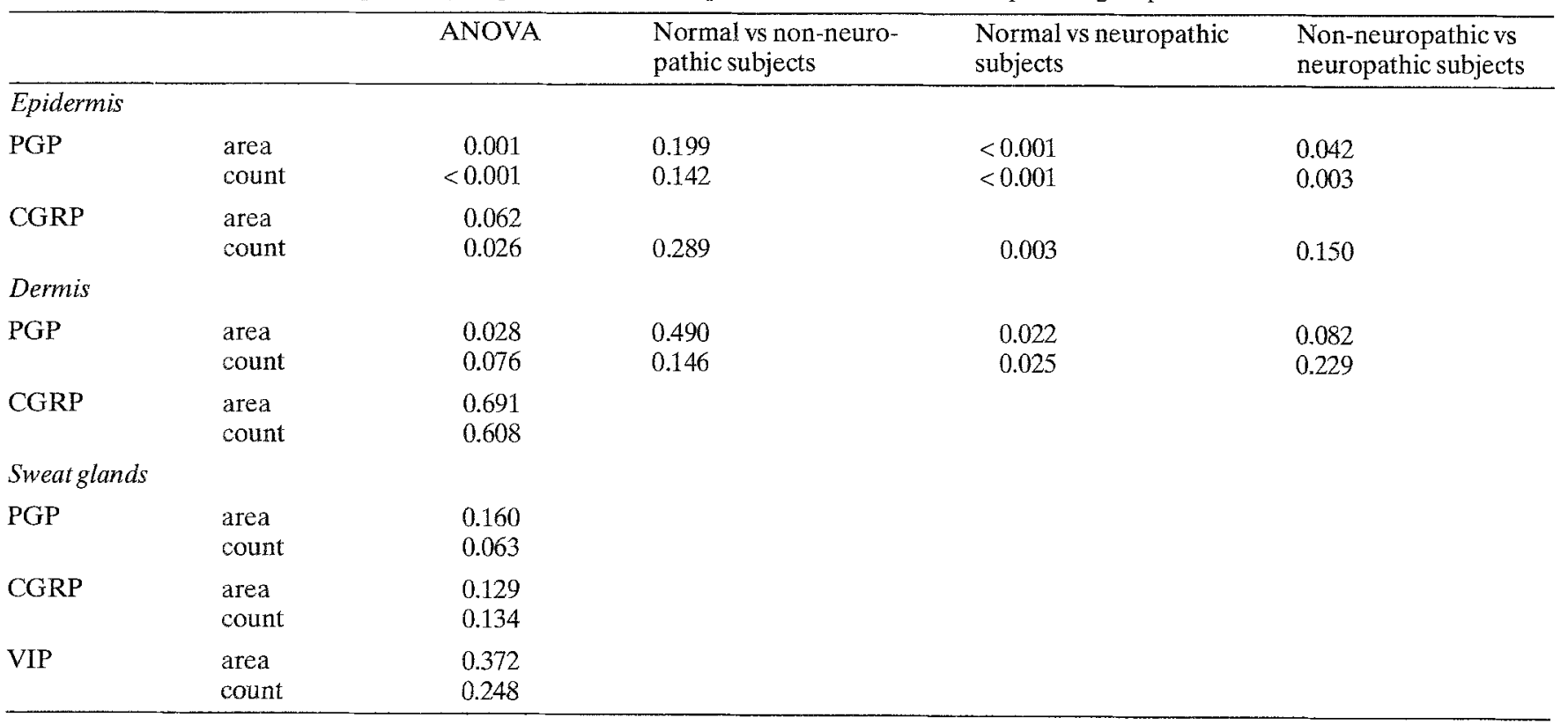

PGP, Protein gene product 9.5; CGRP, calcitonin gene-related peptide; VIP, vasoactive intestinal polypeptide

\section{Histochemical methods}

Consistent orientation of the specimens was maintained from biopsy to analysis. Specimens were fixed in Zamboni's solution for $6 \mathrm{~h}$ at room temperature, then transferred to phosphate-buffered saline (PBS, $0.01 \mathrm{~mol} / \mathrm{l}$ phosphate buffer, $\mathrm{pH} 7.1$, containing $0.15 \mathrm{~mol} / \mathrm{so}$ dium chloride and $15 \%$ sucrose) at $4{ }^{\circ} \mathrm{C}$. Cryostat sections $(10 \mu \mathrm{m})$ were taken up on poly-L-lysine-coated glass slides, allowed to dry for $2 \mathrm{~h}$ at room temperature and then placed in PBS containing $0.2 \%$ Triton X-100 for 30 min. A modified indirect immunofluorescence staining method was used for immunostaining [17], using antisera to PGP 9.5, CGRP and VIP. Following counterstaining with Pontamine sky blue to reduce background autofluorescence, sections were incubated in appropriately diluted primary antiserum for $16 \mathrm{~h}$ at $4^{\circ} \mathrm{C}$, then washed thoroughly for $10 \mathrm{~min}$ in three fresh changes of PBS and re-incubated in the primary antiserum for $3 \mathrm{~h}$ at room temperature. After three further 5 min PBS washes, goat antirabbit fluorescein isothiocyanate conjugate (1:200; Miles Laboratories, Stoke Poges, UK) was applied for $1 \mathrm{~h}$. Slides were washed again for $5 \mathrm{~min}$ in three fresh changes of PBS, mounted in PBS glycerol (1:9 volume/volume) and viewed using a fluorescence microscope.

\section{Immunohistochemical measurements using computerised image-analysis}

The measurement system consisted of a low-light black and white video camera (Panasonic MV 1900, Osaka, Japan) mounted on a Vanox fluorescence microscope (Olympus, London, UK) and linked to an IBAS 2000 image analyser (Kontron, Watford, UK). A 

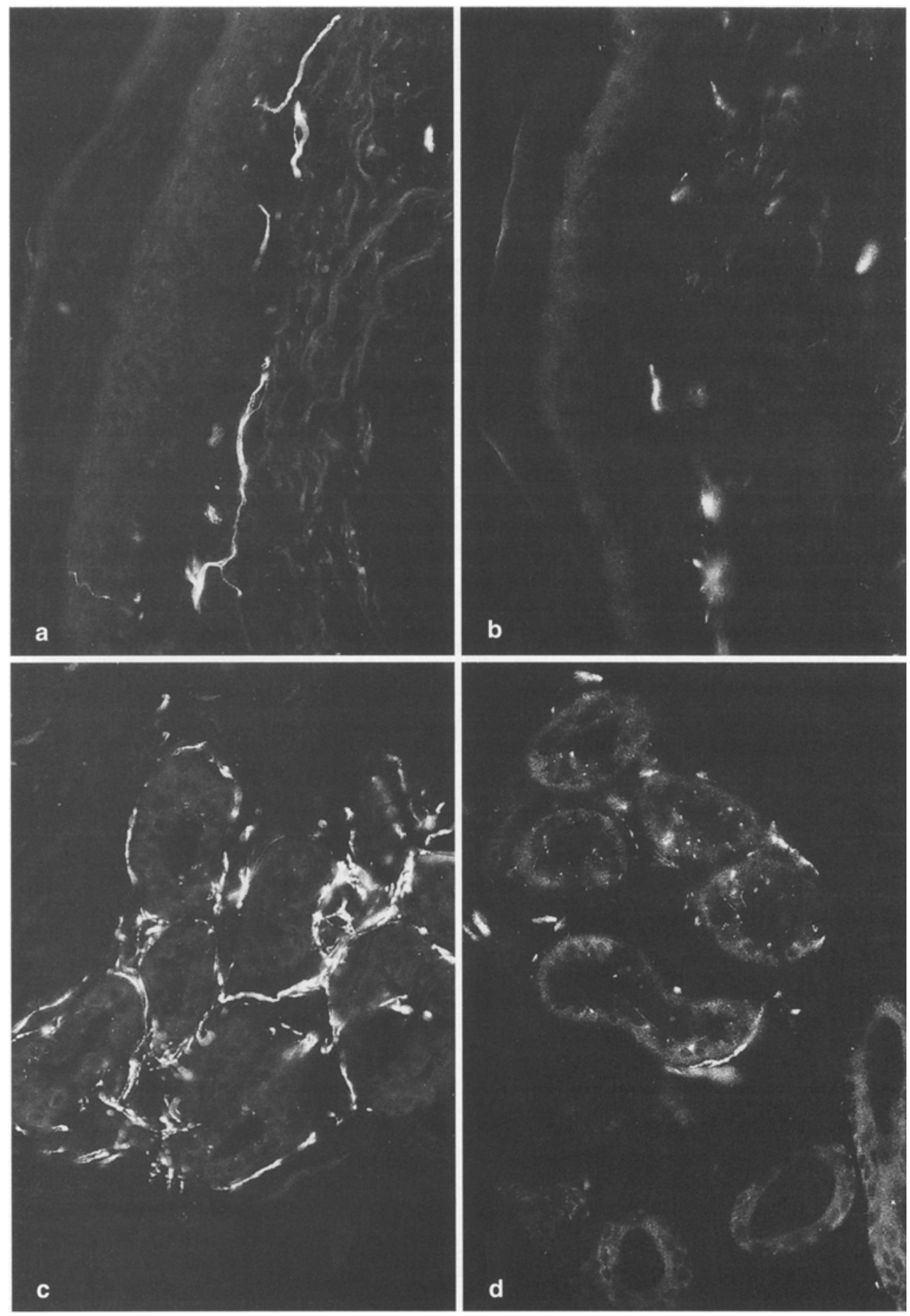

Fig.1 a-d. Protein gene product 9.5-immunoreactive (IR) nerves in the epidermis of (a) normal subject and (b) neuropathic patient, where a marked decrease in reactivity is evident $(\times 281)$. Vasoactive intestinal polypeptide (VIP)IR nerves around a group of sweat glands in (c) normal subject and (d) neuropathic patient $(\times 202)$. While depletion was the most common pattern of VIP-IR in diabetic patients, it was not uniform (see Fig. 3)

long pass filter of approximately $560 \mathrm{~nm}$ was used to reduce autofluorescence. The program for measurement of immunoreactivity was derived from one used in previous studies [18-20]. Ten digitised images of the field to be measured were summed and then averaged to reduce background noise. Possible uneven illumination and shading at the edge of the field was automatically calibrated to the same level of the brightest part of the image, generally the centre of the field. The field image was defined by use of interactively defined measured frames in order to establish precisely the area to be measured (e.g. epidermis and subepidermal plexus), while deleting any unwanted portion of the image present on the screen (e.g. dermal collagenisation, sweat gland granules, artefacts associated with non-specific background fluorescence). The image of the immunostained structures was enhanced by automatic com- puter process to increase the signal:noise ratio; during the process the grey/black tones of the background were darkened, resulting in an improved contrast of the white images corresponding to the immunostained structures. This was particularly important for fine nerve fibres which might otherwise be missed. A background subtraction procedure was performed by taking the maximum grey value of the background as the threshold at which to segregate the image. The grey value for binary thresholding was maintained in the same range for all samples, in order to avoid any threshold bias. The discriminated binary image was finally measured.

For each peptide two random non-consecutive sections were analysed, and in each section three linearly-contiguous fields $(\times 20$ objective magnification) in three skin areas - epidermis and sub- 

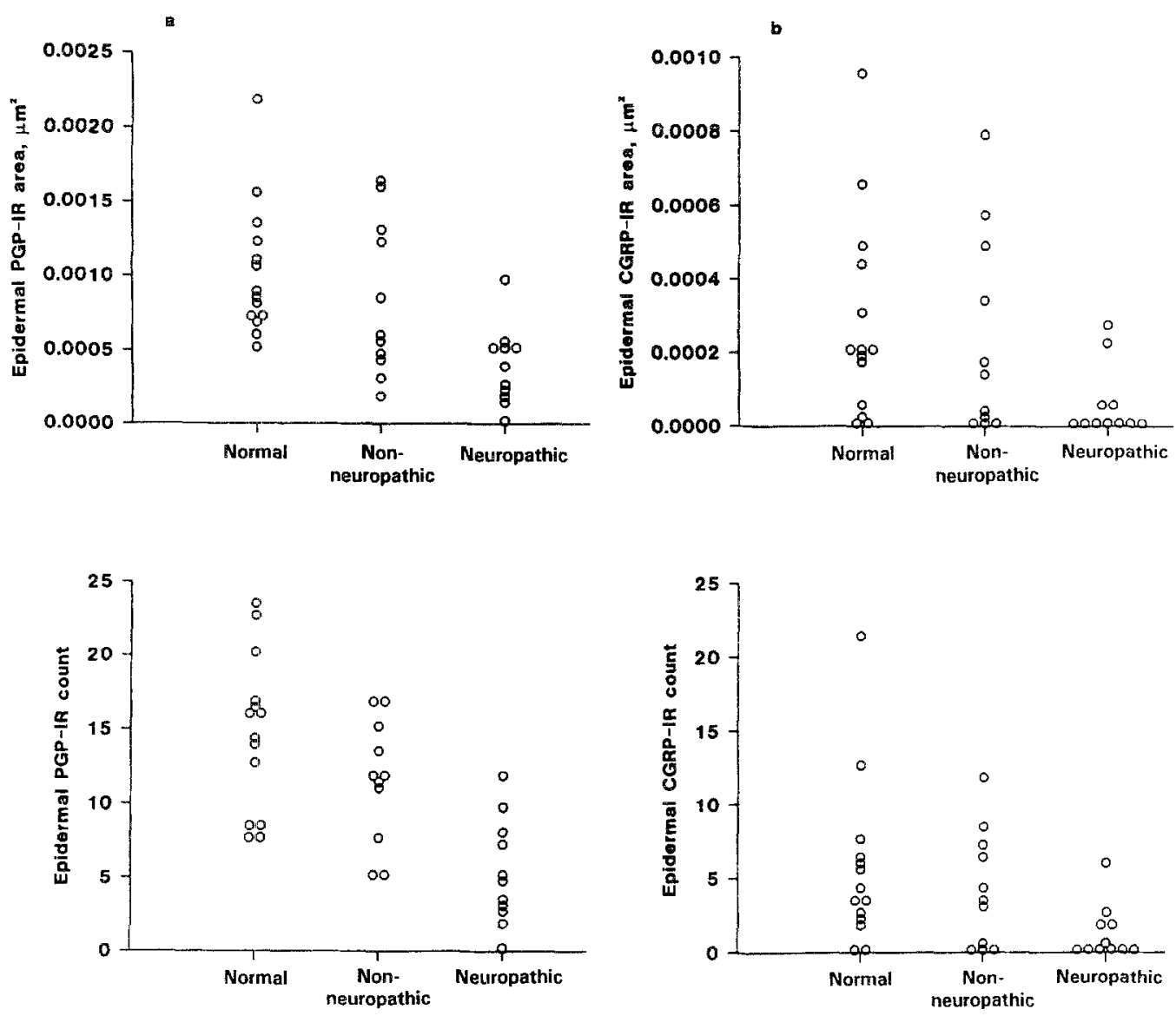

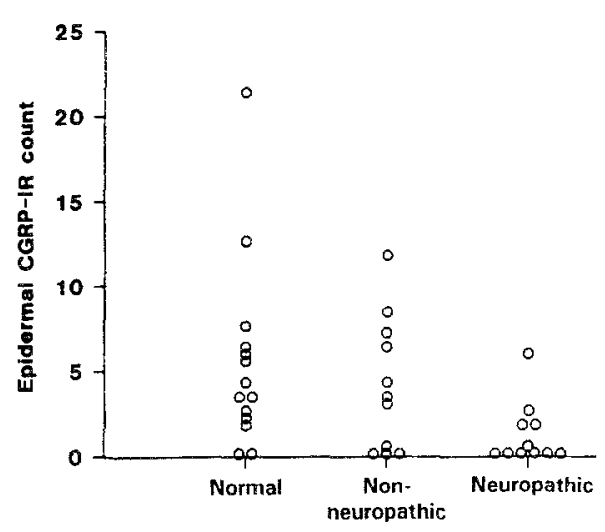

Fig. 2a, b. Dot plots of epidermal nerve areas: (a) Protein gene product 9.5 -immunoreactivity (PGP 9.5-IR) (b) Calcitonin gene-related peptide (CGRP)-IR. Upper panels: IR nerve area; lower panels: nerve counts. Both PGP 9.5and CGRP-IR show progressive reductions through the three groups. The depletion was statistically significant for the neuropathic group vs age-matched normal subjects (PGP 9.5-IR area and count: $p<0.001$; CGRP. IR count: $p=0.003$ ) epidermal plexus, dermis, and sweat glands - were measured. Total field immunofluorescent area $\left(\mu \mathrm{m}^{2}\right)$ and field counts of the immunoreactive nerves were measured in each field. The mean value of the total six counted fields was calculated.

\section{Statistical analysis}

Immunoreactive areas and counts were non-normally distributed; logarithmic transformation normalised the distributions, and analysis of variance (ANOVA) was performed on the logarithmicallytransformed data. Group comparisons were then performed with the Student's $t$-test.

\section{Results}

Tables 3 and 4 show the results for the immunohistochemistry measurements. Photomicrographs of epidermal PGP-IR and sweat gland VIP-IR in normal and neuropathic patients are shown in Figure 1.

\section{Normal subjects}

Qualitatively, the distribution of immunoreactive nerve fibres was similar to that previously reported $[17,21]$. PGPIR was identified in free nerve endings in the epidermis and dermis, and adjacent to hair follicles and sweat glands. Bundles of PGP-IR nerves were seen around blood vessels and arrector pili muscles. CGRP-IR fibres were found predominantly in the dermis; smaller numbers were seen extending into the epidermis and around sweat glands and blood vessels.
Immunohistochemical measurements showed wide variation (Figs. 2 and 3). All normal subjects had measurable epidermal, dermal and sweat gland PGP-IR (Figs. 2 a, $3 a)$. In two specimens no sweat glands were seen.

CGRP-IR was more sparsely distributed in all regions than PGP-IR. Two subjects had absent epidermal CGRPIR (Fig. 2b), but four lacked CGRP-IR in the dermis and one in the sweat glands. All normal subjects had detectable sweat gland VIP-IR (Fig, 3b).

\section{Diabetic patients}

PGP-immunoreactivity. EpidermalPGP-IR showeda progressive reduction in the three groups (Fig. 2a) (area: $p=0.001$, count: $p<0.001$ by ANOVA). The depletion was statistically significant between normal and neuropathic subjects (area and count: $p<0.001$ ), and between nonneuropathic and neuropathic patients (area: $p=0.042$, count: $p=0.003$ ) but not between normal and non-neuropathic subjects (area: $p=0.199$; count: $p=0.142$ ). Dermal PGP-IR area was similar in all groups, though one neuropathic patient had an apparently elevated PGP-IR area. Dermal PGP-IR showed a progressive diminution in the three groups (area: $p=0.028$; count: $p=0.039$ by ANOVA). This was statistically significant between normal and neuropathic subjects (area: $p=0.022$; count: $p=0.025$ ), but not between non-neuropathic and neuropathic subjects (area: $p=0.082$; count: $p=0.229$ ). Sweat gland PGP-IR was similar in the three groups (area: $p=0.160$; 

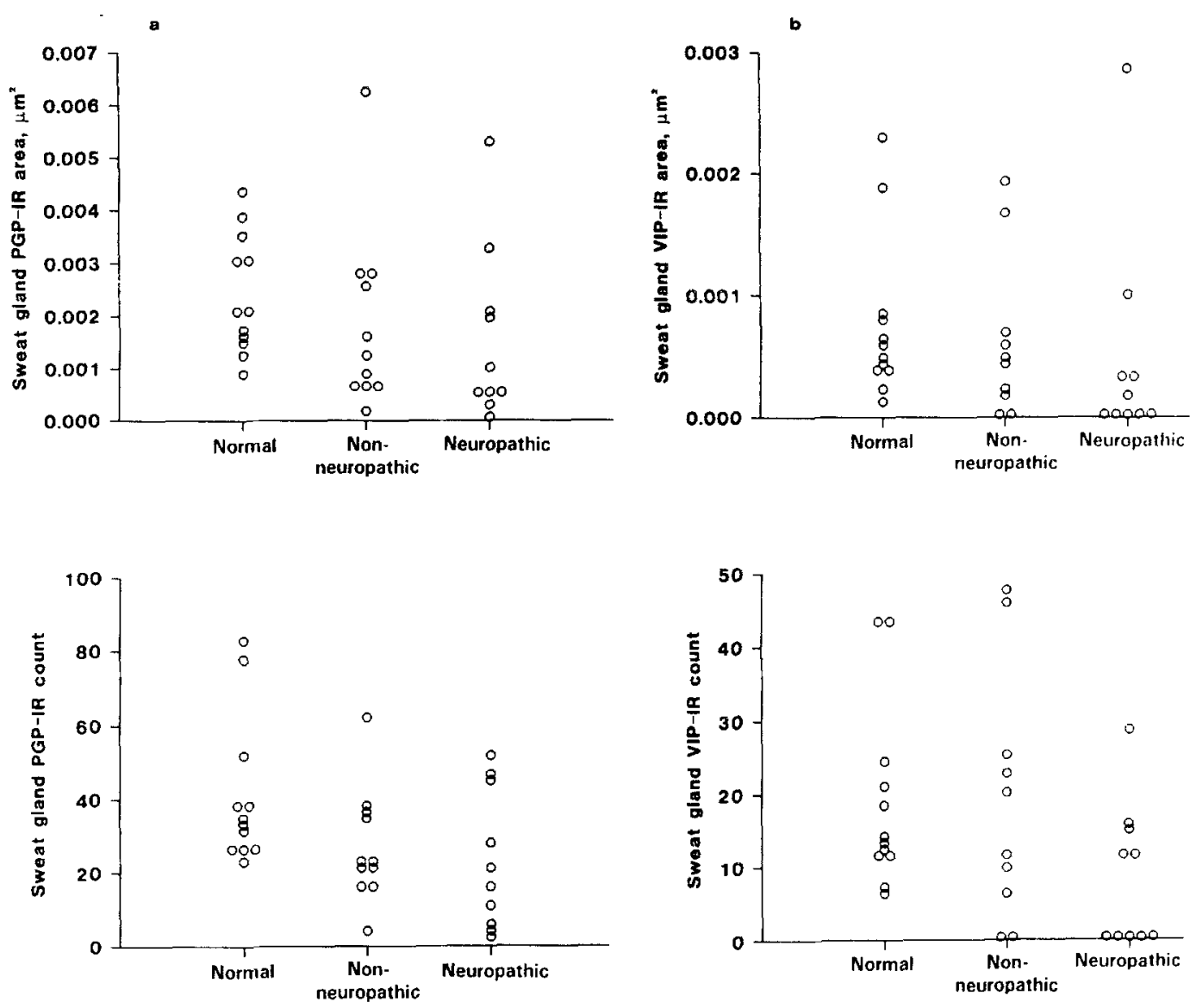

Fig. 3a, b. Dot plots of sweat gland neuropeptide immunoreactivity (IR): (a) Protein gene product 9.5 (PGP 9.5)IR (b) Vasoactive intestinal polypeptide (VIP)-IR. Upper panels: IR nerve area; lower panels: nerve counts. There were no significant differences between the three groups. Two diabetic subjects had elevated PGP 9.5-IR area one neuropathic patient elevated VIP-IR area and two non-neuropathic subjects elevated VIP-IR counts

count: $p=0.272$; Fig. 3 a). One neuropathic and one nonneuropathic patient had elevated IR nerve area.

CGRP-immunoreactivity. As with PGP-IR, epidermal CGRP showed a decrease through the three groups studied, though this was statistically significant for nerve counts $(p=0.026)$ but not for immunoreactive nerve area $(p=0.062)$. Seven neuropathic patients had no detectable epidermal CGRP (Fig. 2b). CGRP-IR count was significantly lower in this group compared with normal subjects $(p=0.003)$ but not with non-neuropathic patients $(p=0.150)$. Dermal CGRP-IR was similar in the three groups (area: $p=0.691$; count: $p=0.608$ ), though one nonneuropathic patient had elevated IR area, and two had increased nerve counts. Sweat gland CGRP-IR also showed no differences between the three groups (area: $p=0.129$; count: $p=0.134$ ).

VIP-immunoreactivity. VIP-IR was absent in all subjects in epidermis and dermis. Sweat gland VIP-IR measurements showed marked variation (Fig. $3 b$ ). Statistically there were no significant differences between the three groups, either in nerve area $(p=0.372)$ or count $(p=0.248)$. VIP-IR depletion was the most common finding (five neuropathic patients, compared with two non-neuropathic patients, had absent VIP-IR) but one neuropathic patient had elevated VIP-IR area, and two non-neuropathic patients had elevated counts.

Physical characteristics (height, weight, and body mass index), age and diabetes duration showed no associations with quantitative immunohistochemical measure- ments. Epidermal and dermal IR correlated negatively with total $\mathrm{HbA}_{1}$ : epidermis PGP-IR area: $r=-0.460$, $p=0.055$, count: $r=-0.553, p=0.05$; dermis PGP-IR area: $r=-0.532, p=0.023$, count: $r=-0.589, p=0.01$ (Fig.4); dermis CGRP-IR area: $r=-0.47, p=0.049$, count: $r=-0.463, p=0.053$.

\section{Associations between immunohistochemical and neurological measurements}

There were no consistent changes in immunohistochemical measurements with thermal or vibratory thresholds, with the common peroneal conduction velocity, or with cardiovascular autonomic function (three tests of heartrate variation and postural systolic and diastolic blood pressure change). None of the results of the sweat tests showed correlations with the epidermal immunohistochemical measurements, and, as expected, the pilocarpineactivated sweatspot density was not related to the histochemical measurements in any skin region. However, significant associations were found between some of the dermal and sweat gland measurements and acetylcholinestimulated sweat output and sympathetic skin response. Basal (non-stimulated) sweating rate (transepidermal water loss rate) and acetylcholine-stimulated sweat output correlated with sweat gland PGP-IR (Fig. 5a) and CGRPIR. The correlations with the sympathetic skin response were different, in that increasing neuropeptide IR was associated with diminished responses (i. e. longer response latencies and smaller response amplitudes) (Fig. 5b). 


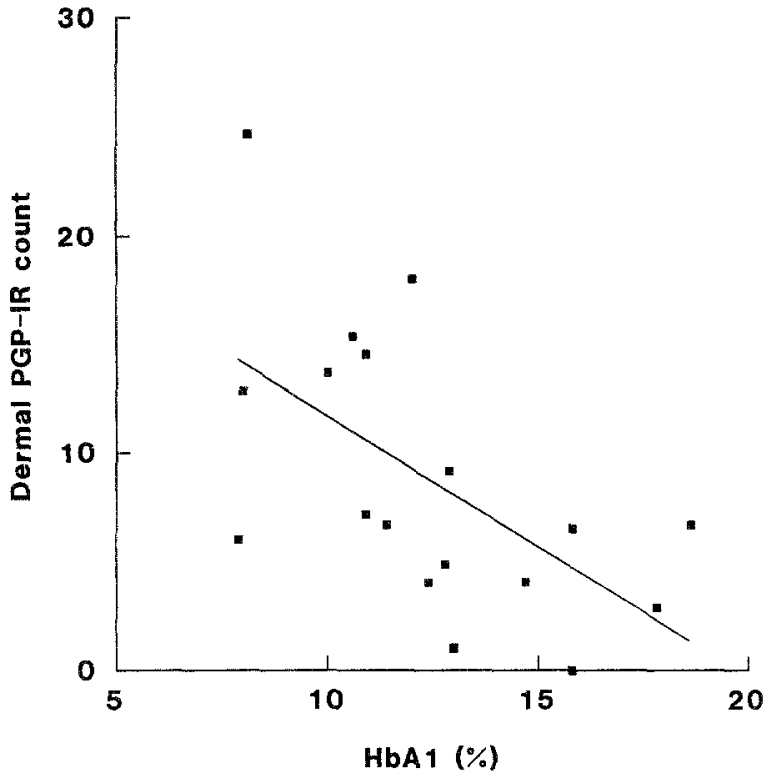

Fig.4. Scatterplot of relationship between total $\mathrm{HbA}_{1}$ and dermal protein gene product (PGP) 9.5-immunoreactivity (IR) count. The linear regression coefficient is $r=-0.59, p=0.01$. Poor diabetic control is therefore associated with diminished IR

\section{Discussion}

Techniques for the histological measurement of peripheral small fibres in diabetes are potentially of great value, as cutaneous afferent sensory and efferent postganglionic sympathetic fibres are thought to be widely involved in diabetic neuropathy. Unmyelinated fibre abnormalities in diabetic sural nerve have been quantified [22], but electron microscopy is required on account of their small size $(0.1-3.0 \mu \mathrm{m}$ diameter) and the analyses are laborious to perform [23]. Immunohistochemical measurement of small fibres in skin biopsies has the advantage of simplicity and potential repeatability. In addition, nerves can be studied at their most distal points, where early changes may be seen, and sensory and autonomic nerve fibres can be separately identified.

While automated image-analysis methods for determination of immunoreactive nerve areas and counts are considered to be more accurate than observer graded assessments, they may be subject to errors resulting from sampling bias, definition of the areas of interest and the method used to determine the threshold at which to segregate the image. We have attempted to minimise such errors by measuring linearly contiguous fields in non-consecutive sections, and by using similar thresholds for all samples. Nevertheless, two-dimensional image analysis of three-dimensional structures may result in over-representation of large nerve fibres, and conversely, omission of very fine fibres from the image. This may not be a significant problem when studying the superficial layers of the skin, where nerve fibres are relatively homogeneous in size, but very small regenerating nerve fibres may still be under-represented. Developments in stereological techniques [24] will be valuable in overcoming any such biases in future studies of the cutaneous innervation in diabetes.
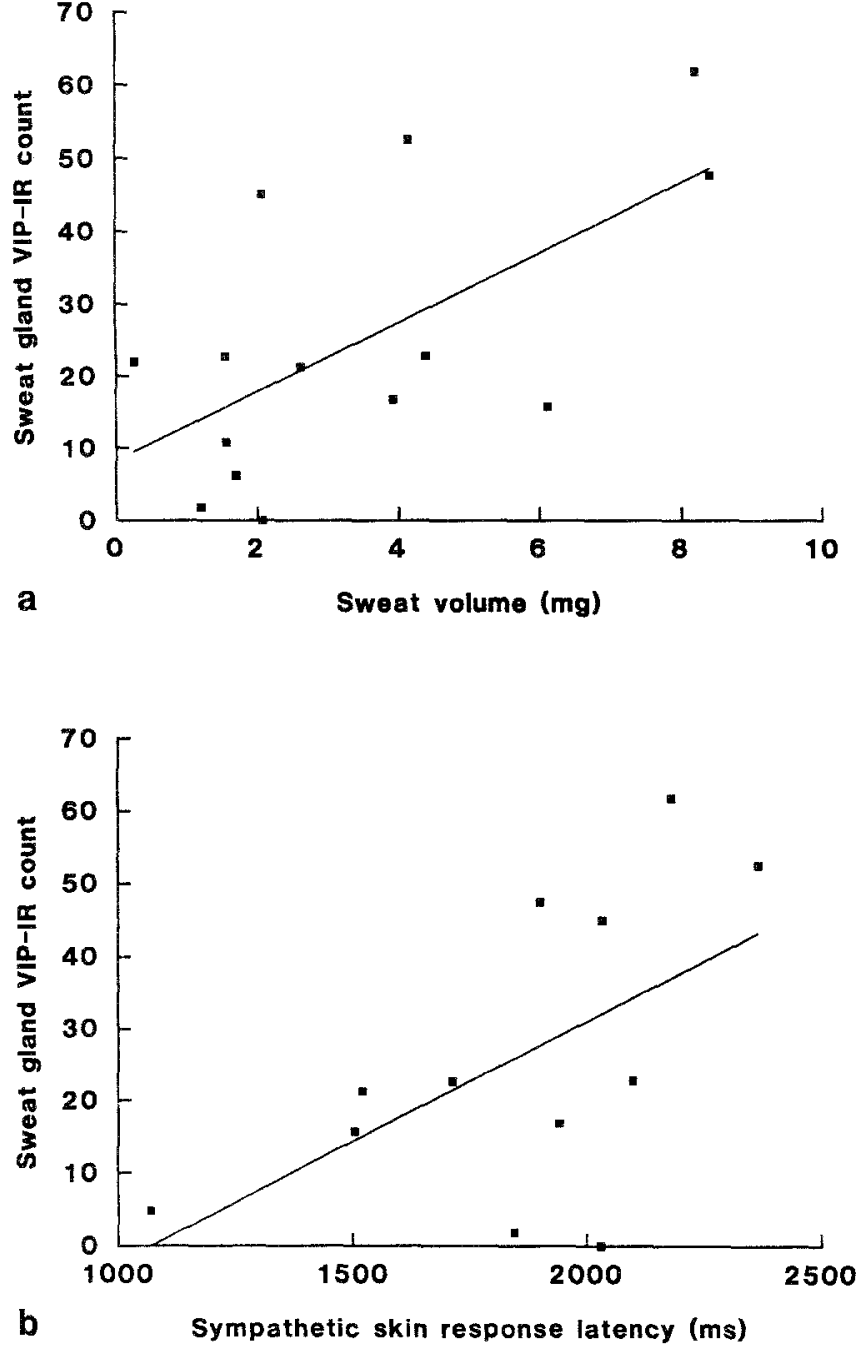

Fig.5a, b. The relationship between sweating function in the feet and quantitative immunohistochemistry. (a) Sweat gland protein gene product (PGP) 9.5-immunoreactivity (IR) nerve counts vs acetylcholine-stimulated sweat output measured with a directreading sudorometer: $r=0.64, p=0.02$; (b) Sweat gland PGP-IR nerve counts vs latency of the sympathetic skin response at the foot: $r=0.72, p=0.01$

The results of the image-analysis method used here for measuring immunoreactive nerve areas and counts are broadly concordant with those of our preliminary study [9] and with the separate analysis of the specimens obtained in the current study [25], both of which used observer grading of immunofluorescent nerve areas. Since we selected young patients, with no clinical evidence of macrovascular disease, the changes seen are unlikely to be due to large-vessel disease. Retinopathy, assessed by direct ophthalmoscopy, was uniformly absent from the "non-neuropathic" patients, suggesting that microvascular disease was also not a contributory factor to nerve depletion, though minor degrees of microangiopathy are clearly not excluded by the absence of clinical retinopathy.

In the epidermis, there was a progressive diminution of the general neuronal marker PGP 9.5 and of the neuropeptide CGRP through the three groups studied, suggesting that depletion of nerves and their associated neuropeptides occurs in parallel in diabetes. Absent epidermal 
CGRP-IR was, however, found more frequently than absent PGP-IR, indicating that CGRP-IR nerve fibres may be particularly susceptible in diabetic neuropathy. The changes did not reach statistical significance when comparing normal and non-neuropathic subjects. This finding is in contrast to the results of Lindberger et al. [10]. Our non-neuropathic subjects were defined using different criteria, emphasising unmyelinated, rather than myelinated nerve fibre function; in addition, though Lindberger et al. [10] studied a smaller number of patients with a greater mean age ( 50 years), their biopsies were slightly more distal than those taken here. The two studies are therefore not strictly comparable.

While there was consistent depletion of PGP 9.5 and CGRP in the epidermis, this was not the pattern seen in the dermis and sweat glands, where, although absent nerves were more frequently found in diabetic compared with normal subjects, there were no statistically significant differences in immunoreactive nerve areas or counts between the groups. As in the epidermis, neuropeptide IR was more frequently absent than PGP-IR; however, in these two regions, there were several patients with increased IR. The reasons for finding both decreased and increased neuropeptide IR in different subjects within these well-defined groups are not known. Increased immunofluorescent nerve areas and counts may be the result of nerve proliferation in some subjects, and there is good experimental evidence for this process, which is probably mediated by nerve growth factor [26]; for example, Kennedy et al. [27] have demonstrated the remarkable regenerative capacity of sudomotor neurons in the mouse after acute denervation. Selective increases in peptidergic nerve terminals in the gut $[28,29]$ and iris [30] of streptozotocin-diabetic rats have been described, while other studies have found neurotransmitter deficits in mesenteric perivascular sympathetic nerves, with no accompanying changes in the total innervation, revealed by immunostaining for PGP 9.5 [31]. There is as yet no coherent explanation for these findings. In the current study, the more marked depletion of epidermal nerves compared with those in the dermis and sweat glands is consistent with a length-dependent neuropathy. The finding of increased immunofluorescence in the dermis and sweat glands may be related to blood flow; these regions, unlike the epidermis, have an abundant capillary supply, and one which may be further increased as a result of sympathetic denervation.

We found consistent negative correlations between $\mathrm{HbA}_{1}$ at the time of the biopsy and most of the immunohistochemical measurements; all the patients studied were in stable diabetic control, and these results confirm that poor glycaemic control is associated with peripheral small-fibre depletion. Further studies are required to demonstrate whether changes in diabetic control are reflected in small-fibre immunohistochemical measurements.

The relationship between the neurophysiological tests and the immunohistochemical findings should be interpreted with caution, as there were small numbers of subjects and the skin biopsies were taken at a site proximal to the one used for the neurological tests. However, while there were no significant correlations with conventional neurophysiological measurements (sensory thresholds, nerve conduction studies and cardiac autonomic tests), there were significant correlations with two of the local tests of sudomotor function. In a related study we have shown a clear dissociation between cholinergic sweat gland activation and neurophysiological tests in neuropathic diabetic subjects [32]. In the test of pharmacologically-stimulated sweat output in response to iontophoresed acetylcholine, increasing sweat output was associated with increased nerve IR, while the amplitude and latency of the sympathetic skin response was inversely correlated with the immunohistochemical measurements. These contrasting results suggest that sweat glands in diabetes may demonstrate pharmacological supersensitivity while their nerve supply is functionally deficient, and both are associated with marked heterogeneity of immunoreactive nerve numbers and areas. These findings underline the importance of assessing local peripheral nerve function and structure in addition to conventional neurophysiological measurements.

In summary, this study has confirmed the practicability of measuring immunofluorescent nerve areas and counts in standardised skin biopsies in diabetic patients. The investigation, while invasive, has a low morbidity and unlike sural nerve biopsy can be repeated. Neuropeptide changes are seen in the absence of clinical macro- and microvascular disease. There are regional variations within the skin, but within regions the general innervation revealed by PGP 9.5 staining appears better preserved than that of neuropeptide IR nerves. As yet unexplained increases in neuropeptide IR have been demonstrated in some diabetic patients. Immunohistochemical measurements reflect some measures of local sweat gland function, but not conventional tests of large- and small-fibre function. This method should prove useful in assessing the structural response of peripheral small fibres to certain therapeutic agents, for example gangliosides [33] and ACTH fragments [34], whose in vitro effects are predominantly the induction of peripheral nerve sprouting.

Acknowledgements. This study was supported by Medical Research Council project grant G8825877N (DML). We thank Mr. D. Rowley and Ms. K.Armstrong for their technical and organisational help, Mr. J.Payne-James FRCS for taking the biopsies, and Dr. S. McHardy-Young for allowing us to study his patients.

\section{References}

1. Bertelsmann FW, Heimans JJ, Van Rooy JCGM, Dankmeijer HF, Visser SL, Van der Veen EA (1987) Peripheral nerve function in patients with painful diabetic neuropathy treated with continuous subcutaneous insulin infusion. J Neurol Neurosurg Psych 50: 1337-1341

2. Ahmed ME, Le Quesne PM (1986) Quantitative sweat test in diabetics with neuropathic foot lesions. J Neurol Neurosurg Psych 49: 1059-1062

3. Watkins PJ, Edmonds ME (1983) Sympathetic nerve failure in diabetes. Diabetologia 25:73-77

4. Lawrence RD (1965) The diabetic life: a concise practical manual, 17th edn. Churchill, London, $p 157$

5. Foreman JC (1987) Peptides and neurogenic inflammation. $\mathrm{Br}$ Med Bull 43: 386-400 
6. Wallengren J, Ekman R, Sundler F (1987) Occurrence and distribution of neuropeptides in the human skin. Acta Derm Venereol (Stockh) 67: 185-192

7. Thompson RJ, Doran JF, Jackson P, Dhillon AP, Rode J (1983) PGP 9.5 - a new marker for vertebrate neurons and neuroendocrine cells. Brain Res 278: 224-228

8. Wilkinson KD, Lee K, Deshpande S, Duerksen-Hughes P, Boss JM, Pohl J (1990) The neuron-specific protein PGP 9.5 is a ubiquitin carboxyl-terminal hydrolase. Science 246: 670-673

9. Levy DM, Karanth SS, Springall DR, Polak JM (1989) Depletion of cutaneous nerves and neuropeptides in diabetes mellitus: an immunocytochemical study. Diabetologia 32: 427-433

10. Lindberger M, Schröder HD, Schultzberg M et al. (1989) Nerve fibre studies in skin biopsies in peripheral neuropathies. I. Immunohistochemical analysis of neuropeptides in diabetes mellitus. J Neurol Sci 93: 289-296

11. Levy D, Abraham R, Reid G (1989) A comparison of two methods for measuring thermal thresholds in diabetic neuropathy. $J$ Neurol Neurosurg Psych 52: 1072-1077

12. Ewing DJ, Clarke BF (1986) Autonomic neuropathy: its diagnosis and prognosis. Clin Endocrinol Metab 15:855-888

13. Bloom S, Till S, Sönksen P, Smith S (1984) Use of a biothesiometer to measure individual vibration thresholds and their variation in 519 non-diabetic subjects. Br Med J 288: 1793-1795

14. Levy DM, Rowley DA, Abraham RR (1991) Changes in cholinergic sweat gland activation in diabetic neuropathy identified by computerised sweatspot analysis. Diabetologia 34: 807-812

15. Levy DM, Reid G, Abraham RR, Rowley DA (1991) Assessment of basal and stimulated sweating in diabetes using a directreading computerized sudorometer. Diabetic Med 8 [Suppl]: S78-S81

16. Shahani BT, Halperin JJ, Boulu P, Cohen J (1984) Sympathetic skin response - a method of assessing unmyelinated axon dysfunction in peripheral neuropathies. J Neurol Neurosurg Psych 47: 536-542

17. Karanth SS, Springall DR, Lucas S et al. (1989) Changes in nerves and neuropeptides in skin from 100 leprosy patients investigated by immunocytochemistry. J Pathol 157: 15-26

18. Cowen T, Alafaci C, Crockard HA, Burnstock G (1987) Origin and postnatal development of nerves showing 5-hydroxytryptamine-like immunoreactivity supplying major cerebral arteries of the rat. Neurosci Lett 78: 121-126

19. Gale JD, Alberts JCJ, Cowen T (1989) A quantitative study of changes in old age of 5-hydroxytryptamine-like immunoreactivity in perivascular nerves of the rabbit. $\mathbf{J}$ Auton Nerv Syst $28: 51-$ 60

20. Terenghi G, Bunker CB, Liu Y-F et al. (1991) Image analysis quantification of peptide-immunoreactive nerves in the skin of patients with Raynaud's phenomenon and systemic sclerosis. J Pathol 164: 245-252

21. Dalsgaard CI, Rydh M, Haegerstrand A (1989) Cutaneous innervation in man visualized with protein gene product 9.5 (PGP 9.5) antibodies. Histochemistry 92: 385-389

22. Behse F, Buchthal F, Carlsen F, Knappeis GG (1975) Unmyelinated fibres and Schwann cells of sural nerve in neuropathy. Brain 98: 493--510
23. Kanda T, Tsukagoshi H, Oda M, Miyamoto K, Tanabe H (1991) Morphological changes in unmyelinated nerve fibres in the sural nerve with age. Brain 114: 585-599

24. Gundersen HJG, Bendtsen TF, Korbo L et al. (1988) Some new, simple and efficient stereological methods and their use in pathological research and diagnosis. APMIS 96: 379-394

25. Levy DM, Springall D, Terenghi G, Reynolds G, Abraham RR, Polak JM (1990) Differential changes in a general neuronal marker and neuropeptides in diabetic skin. Diabetologia 33 [Suppl]: A15 (Abstract)

26. Aberdeen J, Corr L, Milner P, Lincoln J, Burnstock G (1990) Marked increases in calcitonin gene-related peptide-containing nerves in the developing rat following long-term sympathectomy with guanethidine. Neuroscience 35: 175-184

27. Kennedy WR, Sakuta M, Quick DC (1984) Rodent eccrine sweat glands: a case of multiple efferent innervation. Neuroscience 11: 741-749

28. Belai A, Lincoln J, Milner P, Crowe R, Loesch A, Burnstock G (1985) Enteric nerves in diabetic rats: increase in vasoactive intestinal polypeptide but not substance P. Gastroenterology 89: 967-976

29. Belai A, Lincoln J, Milner P, Burnstock G (1988) Progressive changes in adrenergic, serotonergic, and peptidergic nerves in proximal colon of streptozotocin-diabetic rats. Gastroenterology $95: 1234-1241$

30. Crowe $R$, Burnstock $G$ (1988) An increase of vasoactive intestinal polypeptide-, but not neuropeptide Y-, substance P- or catecholamine-containing nerves in the iris of the streptozotocin-induced diabetic rat. Exp Eye Res 47: 751-759

31. Webster GJM, Petch EWA, Cowen T (1991) Streptozotocin-induced diabetes in rats causes neuronal deficits in tyrosine hydroxylase and 5-hydroxytryptamine specific to mesenteric perivascular sympathetic nerves and without loss of nerve fibers. Exp Neurol 113: 53-62

32. Berg TJ, Levy DM, Abraham RR, Reid G (1990) Modulation of methacholine-induced sweating by vasoactive intestinal polypeptide (VIP) and substance P (SP) in diabetic neuropathy. Diabetes 39 [Suppl 1]: 154A (Abstract)

33. Gorio A (1986) Ganglioside enhancement of neuronal differentiation, plasticity and repair. Crit Rev Clin Neurobiol 2: 241-296

34. De Koning P, Verhaagen J, Sloot W, Jennekens FGI, Gispen WH (1989) Org. 2766 stimulates collateral sprouting in the soleus muscle of the rat following partial denervation. Muscle Nerve 12: $353-359$

Received: 9 September 1991

and in final revised form: 29 May 1992

Dr. D.M.Levy

Department of Diabetes, Endocrinology and Metabolism

Radcliffe Infirmary

Woodstock Road

Oxford $\mathrm{OX} 26 \mathrm{HE}$

UK 